\title{
PainVet: escala digital de valoración del dolor en perros
}

\author{
PainVet: digital scale for pain assessment in dogs
}

Castillo-Hidalgo Edy

Universidad Católica de Cuenca ecastilloh@ucacue.edu.ec

Pillaga Gaona Christian Universidad Católica de Cuenca

Rubio Pablo

Universidad Católica de Cuenca

Alvarado Juan

Universidad Católica de Cuenca

Maldonado Manuel Universidad Católica de Cuenca

Revista Cumbres Vol.7 №1

Versión electrónica ISSN 1390-3365

http://investigacion.utmachala.edu.ec/revistas/index.php/Cumbres http://doi.org/10.48190/cumbres.v7n1a6 


\title{
RESUMEN
}

Con el crecimiento exponencial del uso de dispositivos móviles inteligentes, se han desarrollado varias aplicaciones en medicina veterinaria que han contribuido a facilitar el acceso a la información en diferentes ámbitos, en los cuales se incluyen la evaluación del dolor, el cual ha sido una preocupación constante de los profesionales de la salud animal. En el presente trabajo se planteó como objetivo desarrollar e implementar una App digital basados en la escala de Melbourne y Glasgow modificada, desarrollando una base de datos para el sistema operativo Android, obteniendo una escala digital, con un score de 27 puntos, 5 niveles de dolor, y un exportador PDF. Para la verificación de esta aplicación se procedió a cuantificar las variables dependientes que utiliza la escala de Melbourne y Glasgow, evaluando el dolor postquirúrgico en un total de 113 perros, de los cuales, en 43 pacientes se realizaron 208 observaciones en diferentes tiempos por medio de la escala impresa, en tanto que mediante PainVet se observaron y valoraron 70 pacientes, con un total de 197 observaciones, definiendo un intervalo de confianza $(\mathrm{P}<0.05)$. Lo que nos llevó a concluir que PainVet es una herramienta útil, que permite valorar y registrar los niveles de dolor posquirúrgico en caninos; de igual forma se pudo observar que los pacientes con dolor posquirúrgico presentan con mayor frecuencia midriasis, salivación, vómitos y cambios de su estado mental, signos que se convierten en claves en la valoración del dolor posquirúrgico en perros.

Palabras clave: dolor, evaluación, perros, app, smartphone

\begin{abstract}
With the exponential growth of smartphone devices, several applications have been developed in veterinary science that have contributed to facilitate the access to information in different areas, including pain assessment, which has been a constant concern of animal health professionals. The objective of this work was to develop and implement a digital App based on the Melbourne and Glasgow modified scale, rising data for the Android operating system and as result obtain a digital scale, with a score of 27 points, based on 5, levels of pain, and a PDF exporter. For the verification of this application, all the dependent variables used by the Melbourne and Glasgow scale were quantified, in two methods: manual and digital and then compared. 87 post-surgical dogs were used, of which, 43 patients were Manual (printed
\end{abstract}


scale) with a total of 208 observations at different times, while 44 patients were tested with PainVet, with a total of 197 observations, defining a confidence interval $(\mathrm{P}<0.05)$ and a $\mathrm{T}$-test for compare de distribution of results. This led us to conclude that PainVet is a useful tool that allows us to assess and record levels of post-surgical pain in canines. In addition, it was possible to observe that patients with post-surgical pain most frequently present mydriasis, salivation, vomiting, and changes in their mental state, signs that become key in the assessment of post-surgical pain in dogs.

Keywords: Pain, evaluation, dogs, app, smartphone

\section{INTRODUCCIÓN}

El dolor es una experiencia consciente, en la que influyen recuerdos, emociones e involucra factores genéticos y cognitivos entre otros (Pérez et al. 2018). Para la IASP (International Association for the Study of Pain) es una experiencia sensorial aversiva causada por un daño real capaz de provocar reacciones motoras y vegetativas de evasión características para cada especie (Zysman, 2012), esta definición considera que el dolor no es una experiencia puramente nociceptiva, sino que incluye componentes emocionales y subjetivos inseparables de la sensación dolorosa (Montes, 2017), consecuentemente el dolor es una sensación no placentera, producido por procesos que dañan o son capaces de dañar los tejidos (Zegarra, 2007) y que se miden en la magnitud de ese probable perjuicio (Gutiérrez et al., 2018), por lo tanto, en la actualidad no existen duda que los animales son capaces de sentir dolor y los mecanismos nerviosos que participan en las diferentes etapas de la respuesta a estímulos dolorosos son esencialmente similares en los animales y en el hombre (Otero, 2005), debiendo considerarse que la incapacidad de comunicarse de ningún modo anula la posibilidad de sentir dolor (Grimm et al., 2013).

La nocicepción es la recepción de señales procedentes de la activación de nociceptores que detectan estímulos que dañan los tejidos (Grimm et al., 2013). Mediante este proceso los estímulos nocivos son captados y transducidos por los nociceptores, que proyectan información aferente al SNC, en donde la integración de las funciones del tálamo, la corteza somatosensorial, las estructuras reticulares y las límbicas, dan lugar a la percepción del dolor (Morales, 2016). Estímulos que tienen en común la propiedad de amenazar la integridad del cuerpo (Plaghki et al., 2018), por tanto, es una experiencia consiente influenciada por la memoria, las emociones, factores genéticos y cognitivos (Aliaga et al., 2015).

La activación de los nociceptores normalmente se realiza a través de diversos mediadores que ejercen una acción excitatoria o inhibitoria de las terminaciones nerviosas aferentes (Romera et al., 2000). En este contexto la activación de las fibras A- $\delta$ mielinizadas se asocia con el dolor agudo, y son 
de alta conducción 5 a $30 \mathrm{~m} / \mathrm{seg}$ y las fibras C, no mielinizadas se asocian al dolor lento tipo quemada (Gutiérrez et al., 2018). Los nociceptores además liberen mediadores cono la sustancia $\mathrm{P}$, que produce vasodilatación, desgranulación de mastocitos e induce la producción y liberación de mediadores inflamatorios (Del Arco, 2015).

El dolor se puede clasificar de acuerdo a su duración, origen, patogenia y características (Zegarrag, 2007). El dolor nociceptivo, ocurre cuando los receptores neurales periféricos son activados por estímulos nocivos como incisiones quirúrgicas, traumatismos, calor y frio; el dolor inflamatorio resulta de la activación del sistema inmunológico en respuesta a una lesión o infección, en tanto que el dolor patológico o también llamado desadaptativo, ocurre cuando el dolor es amplificado y sostenido por cambios moleculares, celulares y micro anatómicos, denominados colectivamente hipersensibilización periférica y central (Hellyer et al., 2007). El dolor agudo es además un mecanismo biológico de alarma de primera línea, cuya función es poner en alerta al organismo frente a estos estímulos nocivos (Pérez y Castañeda, 2012).

Al ser el dolor una experiencia individual y subjetiva, no existe método científico que lo haga "medible" (Vicente et al., 2018). En la actualidad se han publicado muchos métodos de puntuación diferentes que incluyen variables fisiológicas y conductuales, pero pocos se han validado de manera rigorosa. La valoración del dolor en animales es en particular compleja porque deben considerarse diferencias de sexo, edad, especie, raza, cepa y ambiente (Grimm et al., 2013). La medición subjetiva es la forma más frecuentemente utilizada para medir el dolor. Pudiendo ser dividido en tres categorías: Métodos unidimensionales, métodos duales y métodos multidimensionales (Serrano-Atero et al., 2002). Destacándose entre ellos los siguientes:

Escala de valoración numérica (NRS): En esta escala el observador registre una puntuación subjetiva para diferentes intensidades de dolor (Mathews et al., 2014), siendo una de las más utilizadas en el ámbito clínico como en la investigación (Malouf y Baños, 2006).

Escala analógica visual (VAS): es un sistema de puntuación semi-objetivo utilizado para cuantificar la intensidad del dolor (Gaynor y Muir, 2009), es típicamente una línea recta horizontal de $100 \mathrm{~mm}$ de longitud, que describe la intensidad del dolor en scores: a la izquierda la valoración de menor intensidad del dolor y a la derecha el de mayor intensidad (Heinze et al., 2001).

Escala descriptiva simple: Esta presenta segmentos con un respectivo nivel y nombre como son: no dolor, leve, moderado, severo, muy severo y máximo dolor posible (Eizaga y García, 2015).

Escala de Melbourne: es una escala basada en respuestas comportamentales y fisiológicas específicas, que incluyen descriptores múltiples en seis categorías. Dichas categorías tienen observaciones de comportamiento que limitan la interpretación y la propensión del observador, además de evaluar los cambios en el comportamiento o la conducta, lo que aumenta la sensibilidad de la escala. Sin embargo, hasta ahora, su validación aún es limitada (Heinze Pohl et al., 2001). 
Escala de Glasgow: Comprende 47 palabras bien definidas y colocadas en una categoría fisiológica y siete categorías conductuales. Estas últimas comprenden valoraciones de postura, comodidad, vocalización, atención a la herida, talante, respuesta a personas, movilidad y respuesta al tacto. Cada descriptor está bien definido para evitar interpretaciones erróneas. La valoración incluye tanto observación desde cierta distancia, así como la interacción con el paciente, p. ej., palpación de la herida. En todas las escalas son necesarias valoraciones frecuentes porque el dolor no es un proceso estático y deben determinarse los beneficios de la intervención con analgésicos (Grimm et al., 2013).

\section{MATERIALES Y MÉTODOS}

En la presente investigación se desarrolló una aplicación que permite medir el dolor post quirúrgico en perros, basados en la escala de Melbourne y Glasgow modificada, la misma que fue alojada en los sistemas operativos Android, contado para ello con la colaboración de la empresa Signare Software Solutions en la parte informática, obteniéndose una escala digital de medición del dolor postquirúrgico en perros, con un score de 27 puntos, proporcionando puntajes individuales para cada nivel de dolor, diferenciándose 5 niveles e igual número de puntajes que van de 0 a 6 (sin dolor); 7 a 11 (dolor de nivel bajo); 12 a 16 (nivel de dolor de intensidad media); 17 a 20 (dolor de nivel alto) y; 21 a 27 (máximo nivel de dolor), sugiriéndonos al final del test una recomendación de rescate analgésico con niveles de dolor superiores a 7, contando además con un exportador en archivo PDF de la tabla de evaluación, basados en la escala de Melbourne y Glasgow modificada.

En la determinación del tamaño de la muestra se tomó en cuenta a todos los pacientes que asistieron a la clínica veterinaria de la Universidad Católica de Cuenca y que fueron sometidos a diferentes intervenciones quirúrgicas en los meses de septiembre a diciembre del 2019.

\section{Verificación}

Para verificación del presente estudio se trabajó al inicio con 43 perros domésticos con un peso promedio de $13.2 \mathrm{~kg}$ y una edad promedio de 3 años que fueron intervenidos quirúrgicamente en cirugías de carácter reproductivo (OVH/ Orquiectomía), a los cuales se les evaluó individualmente el dolor, en 208 observaciones posteriores a la misma y que fueron registradas en la ficha impresa de la escala de dolor de Melbourne y Glasgow. De forma posterior y mediante el uso de la aplicación PainVet, se evaluaron 44 perros domésticos distintos con un total de 124 observaciones, sometidos al mismo tipo de intervención quirúrgica y los cuales presentaron un peso promedio de $14,1 \mathrm{~kg}$ y una edad promedio de 2,95 años. 
Las mediciones se realizaron con un promedio de 15 minutos posterior a la intervención y posteriormente se realizaron mediciones sistemáticas a la 1 , 2, 4 y 6 horas después a la intervención, evidenciándose mayor facilidad en el manejo de la escala digital, por parte del operario quienes requirieron únicamente su dispositivo electrónico para su valoración.

Se estableció el Intervalo de Confianza (95\%) de las evaluaciones de dolor posquirúrgico para los pacientes que fueron evaluados estableciéndose un valor promedio de dolor de 12,77 con un valor inferior de 12,09 y un valor superior de 13,47; observándose que las valoraciones de signos como la midriasis presentan el mayor peso $(37,66 \%)$ en la valoración final del índice de dolor, así como el estado mental influye altamente $(15,58 \%)$ en el valor final del índice del dolor, siendo estos los indicadores que más aportan al dolor posquirúrgico en animales que se sometieron a intervenciones quirúrgicas reproductivas, tal como se observa en la Tabla 1.

Tabla 1. Factores que determinan la valoración del dolor.

\begin{tabular}{|l|l|l|l|}
\hline \multicolumn{4}{|c|}{ INTERVALO DE CONFIANZA 95\% } \\
\hline & Promedio & Bajo & Alto \\
\hline Postura & 1.28 & 1.06 & 1.51 \\
\hline Aparaciencia & 1.30 & 1.04 & 1.56 \\
\hline Midriasis & 4.64 & 4.47 & 4.81 \\
\hline Vocal & 0.89 & 0.60 & 1.19 \\
\hline Estado mental & 1.76 & 1.53 & 1.99 \\
\hline Marcha & 1.40 & 1.20 & 1.60 \\
\hline Palpación & 1.47 & 1.21 & 1.73 \\
\hline
\end{tabular}

Para comparar la validez y confianza de la aplicación con respecto a la escala manual se analizó la curva de distribución normal para cada momento (APP vs Manual). La curva de los datos obtenidos de forma Manual fue leptocurvica $(0,63)$ y la asimetría fue ligeramente negativa $(-0,21)$. La curva de los datos obtenidos con la App fue platocurvica (-1) y la asimetría fue ligeramente positiva $(0,19)$. Esta distribución demuestra que la APP albergo un mayor rango de valores y los datos que se recogieron de forma Manual, ligeramente se desviaron a valores inferiores con respecto a la media.

La validez de los datos se representan en la gráfica de la Distribución Marginal de las respuestas recogidas en la APP frente a los datos levantados de forma Manual (Figura 1), y se evaluó la precisión de la distribución Normal, de la curva con una prueba de $\mathrm{T}$ para evaluar la diferencias entre observaciones $(\mathrm{P}=0,83)$ evidenciándose la confiabilidad de los valores. 
Figura 1. Comparación de la Distribución Marginal de la APP vs datos Manuales

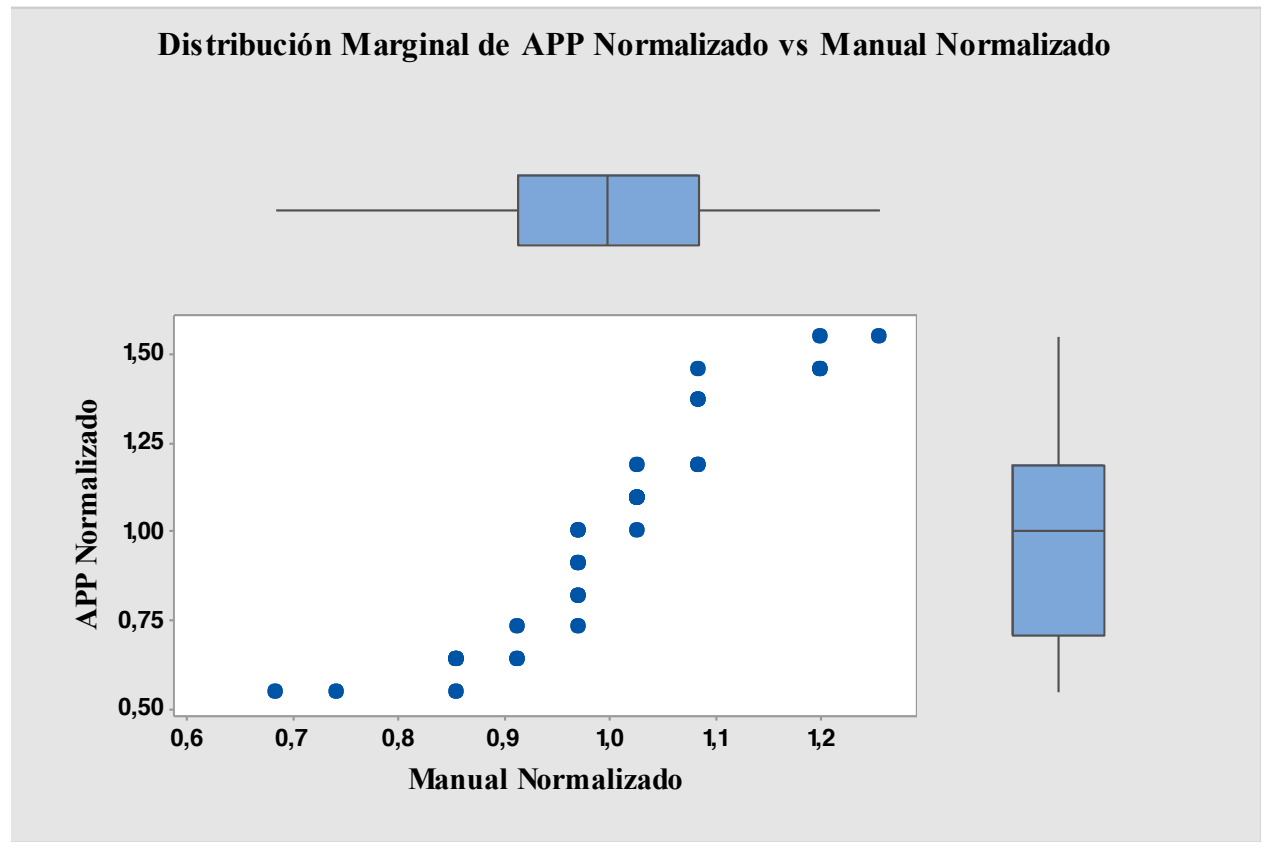

\section{RESULTADOS Y DISCUSIÓN}

Para evaluar el dolor, Vicente et al., (2018) realizó una búsqueda de distintas escalas y cuestionarios que han sido utilizados dentro de estudios clínicos validados para valorar el dolor, considerando el dolor como un objeto de valoración y calificación en diversos países en función de sus baremos, demostrando en su comparativa de escalas y cuestionarios la complejidad de la evaluación del dolor, puesto que se trata de una experiencia individual subjetiva, procedimiento que coincide con el trabajo realizado en la presente investigación, en la cual también se realizó una búsqueda de los diferentes cuestionarios y escalas de valoración del dolor con el fin de poder digitalizar la ficha clínica.

Miró et al. (2017), desarrollaron una aplicación móvil, denominada Painometer v2 ${ }^{\circledR}$, que sirve para medir y registrar el dolor a lo largo del tiempo, en la que incluyen cuatro escalas para medir la intensidad del dolor en humanos. La aplicación es compatible con el sistema operativo Androide y de descarga gratuita desde el Play Store de Google. Características que son seguidas también en el desarrollo de PainVet, la misma que fue desarrollada para la evaluación del dolor posquirúrgico en perros, incluyendo 5 escalas para medir la intensidad del dolor, mientras Painometer v2 ${ }^{\circledR}$, fue sometida a pruebas de fácil uso de forma inicial en un primer ciclo, en el cual tuvo la 
participación de 32 personas, 19 eran profesionales de la salud ( $\overline{\mathrm{x}}$ edad $=$ 31,2 años;) y 13 eran no profesionales de la salud (pacientes potenciales) ( $\overline{\mathrm{x}}$ edad $=17,9$ años). La aplicación PainVet fue sometida a pruebas de aplicabilidad con la participación de médicos veterinarios y estudiantes de la carrera.

\section{CONCLUSIONES}

Los resultados obtenidos a través de la app PainVet, en la valoración del dolor post quirúrgico en perros no muestran variabilidad en su interpretación independiente del tipo de cirugía a la que haya sido sometido el paciente, mostrado una alta fiabilidad en los niveles de dolor obtenidos a través de sus diferentes scores independiente del sexo, edad y raza del paciente, al igual que de la administración de drogas con acción analgésica pre o transquirúrgica, además de demostrar una rápida aceptación por parte de los médicos veterinarios, debido a su adaptabilidad y fácil manejo frente a las fichas físicas, dando con esto respuesta a las necesidades de monitorización del paciente.

\section{Agradecimientos}

Dejamos constancia de nuestro agradecimiento a la Clínica Veterinaria y Jefatura de Comunicación - Área de Marketing \& Publicidad de la Universidad Católica de Cuenca, así como a la empresa Signare Software Solutions.

\section{REFERENCIAS BIBLIOGRÁFICAS}

Aliaga Fron, L., Agra Montava, I., Bainac Albadalejo, L. A., Castillo Pérez, C., Català Puigbò, E., Díaz Jové, R., Durán Olivé, C., Fariñas Balaguer, Ó., Ferrándiz Mach, M., García-Muret, A., Genové Cortada, M., Gil de Bernabé Sala, M. . À., Gómez González, A., Hernández Ontiveros, H., \& Illa Ribera, S. (2015). Manual de Tratamiento del dolor (E. Catala, M. Ferrándiz, \& M. Genové (eds.); 3era ed.). Comgrafic.

Del Arco, J. (2015). Curso Básico Sobre el Dolor. Farmacia Profesional, 29(02139324), 36-43. https://www.elsevier.es/es-revista-farmacia-profesional-3-articulo-curso-basico-sobre-dolor-tema-X0213932415727485

Eizaga Rebollar, R., \& García Palacios, M. V. (2015). Escalas de Valoración de Dolor.

Gaynor, J., \& Muir, W. (2009). Manual de controle da dor em Medicina Veterinária (MedVet (ed.); 2da ed.).

Grimm, K., Lamont, L., \& Tranquili, W. (2013). Manual de anestesia y analgesia en pequeñas especies. El manual moderno.

Gutiérrez Blanco, E., Acevedo Arcique, C. M., Aguilar Caballero, A., \& Ortega Pacheco, A. (2018). Manejo del dolor en el paciente con trauma. Actualidad En Medicina Veterinaria y Zootecnia México, 21(2007-5952), 5-12. 
Heinze Pohl, V., Bonfim Carregaro, A., Lopes, C., Garlet, C., \& Santana Marquez, J. (2001). Correlação entre as escalas visual analógica, de Melbourne e filamentos de Von Frey na avaliação da dor pós-operatória em cadelas submetidas à ovariossalpingohisterectomia. Ciencia Rural, 41, 154-159.

Hellyer, P., Rodan, I., Brunt, J., Downing, R., Hagedorn, J. E., \& Ann Robertson, S. (2007). AAHA/AAFP pain management guidelines for dogs and cats. Journal of Feline Medicine and Surgery, 9, 466-480. https://doi. org/10.1016

Malouf, J., \& Baños, J. (2006). La evaluación clínica del dolor. Revista Clínica Electrónica En Atención Primaria, 10, 1-8.

Mathews, K., Kronen, P. W., Lascelles, D., Nolan, A., Robertson, S., Steagall, P. V., Wright, B., \& Yamashita, K. (2014). Guidelines for recognition, assessment and treatment of pain: WSAVA Global Pain Council members and co-authors of this document:. The Journal of Small Animal Practice, 55(6), E10-68. https://doi.org/10.1111/jsap.12200

Miró, J., de la Vega, R., Roset, R., Castarlenas, E., \& Sánchez, E. (2017). Painometer v2®: Una aplicación móvil certificada para monitorizar a los pacientes con dolor. Revista de La Sociedad Española Del Dolor, 25(2), 112-120. https://doi.org/10.20986/resed.2017.3555/2016

Montes Pérez, A. (2017). Fisiopatología del dolor crónico: de la periferia al cortex cerebral. Vías de transmisión. Sistemas moleculares. Neurotransmisores. Neuromoduladores. http://www.scartd.org/arxius/fisiodolor02. pdf

Morales Vallecilla, C. A. (2016). Bases para el manejo del dolor en perros y gatos (1st ed.). Universidad de Antioquia.

Otero, P. (2005). Fisiopatología y terapéutica del dolor aplicadas a la analgesia en los animales. Revista Argentina de Anestesiología, 63, 339-348.

Pérez Ruiz, A. O., Jiménez Gutiérrez, M. B., \& Vega Cisneros, L. (2018). Regiones del encéfalo vinculadas a la interpretación del dolor. Revista Habanera de Ciencias Médicas, 17(1729-519X), 386-395.

Pérez, T., \& Castañeda, J. (2012). Fisiopatología del dolor agudo: alteraciones cardiovasculares, respiratorias y de otros sistemas y órganos. Revista Cubana de Anestesiología y Reanimación, 11(1), 19-26. http://scielo.sld. cu/scielo.php?script=sci_arttext\&pid=S1726-67182012000100004\&ln$\mathrm{g}=\mathrm{es} \& \mathrm{nrm}=$ iso

Plaghki, L., Mouraux, A., \& Le Bars, D. (2018). Fisiología del dolor. EMC - Kinesiterapia - Medicina Física, 39(1), 1-22. https://doi.org/10.1016/S12932965(18)88603-0

Romera, E., Perena, M., Perena, M., \& Rodrigo, M. (2000). Fisiopatología del dolor. Revista de La Sociedad Española Del Dolor, 7(2), 11-17.

Serrano-Atero, M., Caballero, J., Cañs, A., García-Saura, P., Serrano-Álvarez, C., \& Prieto, J. (2002). Valoración del dolor (I). Revista de La Sociedad Española Del Dolor, 9, 94-108. 
Vicente Herrero, M. T., Delgado Bueno, S., Bandrés Moyá, F., Ramírez Iñiguez de la Torre, M. V., \& Capdevila García, L. (2018). Valoración del dolor. Revisión Comparativa de Escalas y Cuestionarios. Revista de La Sociedad Española Del Dolor, 25(4), 228-236. https://doi.org/10.20986/resed.2018.3632/2017

Zegarra Piérola, J. W. (2007). Bases fisiopatológicas del dolor. Acta Médica Peruana, 24, 105. https://doi.org/1728-5917

Zysman, M. (2012). Dolor en caninos y felinos. Infovet, 17(120), 7.

\section{Cumbres}

